\title{
Determination of the neutrino mass hierarchy with a new statistical method
}

\section{S. Dusini}

INFN - Sezione di Padova, 35131 Padova, Italy

E-mail: stefano.dusiniepd.infn.it

\section{Stanco}

INFN - Sezione di Padova, 35131 Padova, Italy

E-mail: luca.stancodpd. infn. it

\section{Tenti*}

INFN - Sezione di Bologna, 40127 Bologna, Italy

E-mail: matteo.tentiabo.infn.it

\begin{abstract}
Nowadays neutrino physics is undergoing a change of perspective: the discovery period is almost over and the phase of precise measurements is starting. The three-flavour oscillation neutrino framework is strengthening well. In this framework a new method has been developed to determine the neutrino mass ordering, one of the still unknown and most relevant parameters. The method is applied to the 2015 results of the NOvA experiment for $v_{\mu} \rightarrow v_{e}$ appearance, including the treatment of the systematic errors. A substantial gain in significance is obtained compared to the traditional $\chi^{2}$ approach. Assuming the number of the 2015 observed $v_{e}$ events scales with the exposure, an increase of only a factor three in exposure would exclude the inverted hierarchy at more than $95 \%$ C.L. over the full range of the CP violating phase
\end{abstract}

XVII International Workshop on Neutrino Telescopes

13-17 March 2017

Venezia, Italy

${ }^{*}$ Speaker. 


\section{Introduction}

Nowadays, the phenomenology of the neutrino oscillations is coherently described by the three flabours neutrino model, namely, the mixing of 3 weak neutrino eigenstates with 3 not-degenerate mass neutrino eigenstates. Among the few parameters of the three flavours neutrino model to be determined there is the ordering of the neutrino mass eigenvalues, defined as the sign of the difference of the squared masses of $v_{3}$ and $v_{1}\left(\Delta \mathrm{m}_{31}^{2}=\mathrm{m}_{3}^{2}-\mathrm{m}_{1}^{2}\right)$. Two neutrino mass hierarchies can occur: normal hierarchy $(\mathrm{NH})$ when $\Delta \mathrm{m}_{31}^{2}>0$ or inverted hierarchy (IH) in the opposite case. The neutrino mass ordering (MO) is of utmost importance to provide inputs for future studies and experimental proposals, to finally clarify whether we need new projects at all, and to constrain analyses in other fields such as cosmology and astrophysics.

\section{Standard techniques for MO}

All the methods developed so far for establishing whether MO is normal or inverted are based on $\chi^{2}$ evaluation. Given the current uncertainties of the oscillation parameters [1] from few percents to more than $10 \%$, the computation of the difference of the $\chi^{2}$ best fits for $\mathrm{NH}$ and $\mathrm{IH}$ is performed. These analyses use the test statistic

$$
\Delta \chi^{2}=\chi_{\min }^{2}(I H)-\chi_{\min }^{2}(N H)
$$

where the two minima are evaluated spanning the uncertainties of the three neutrino oscillation parameters, namely $\Delta \mathrm{m}_{21}^{2}, \pm \Delta \mathrm{m}_{31}^{2}, \theta_{12}, \theta_{23}, \theta_{13}$ and $\delta_{\mathrm{CP}}$. The parameters $\theta_{i j}(i, j=1,2,3)$ are the mixing angles in the standard parametrization and $\Delta \mathrm{m}_{21}^{2}=\mathrm{m}_{2}^{2}-\mathrm{m}_{1}^{2}$. The statistical significance in terms of standard deviations is computed as $\sqrt{\Delta \chi^{2}}$. The limits of such procedures are well known $[2,3]$. In particular, the significance corresponds only to the median expectation and does not consider the intrinsic statistical fluctuations. Thus, errors of type I and II should be taken into account when comparing the probability density functions of $\Delta \chi^{2}$. As a consequence the corrected significance is lower and more $\sigma$ 's are needed to reach a robust observation. Despite these caveats no alternative test statistic has been outlined so far.

\section{A new techniques for MO}

A new test statistic is defined following a Bayesian approach developed in a frequentist way. For this purpose, the NOvA 2015 results [4] are used as working example. They were re-obtained with GLoBES package [5,6]. Fig. 1 shows the number of predicted oscillated $v_{\mu} \rightarrow v_{e}$ events plus the expected background as a function of $\delta_{\mathrm{CP}}$ for the $\mathrm{IH}$ and $\mathrm{NH}$ hypotheses. As can be noted, no discrimination between $\mathrm{IH}$ and $\mathrm{NH}$ can be achieved if the $\Delta \chi^{2}$ minimization is performed.

The new technique, based on a new test statistic that properly weights the intrinsic statistical fluctuations of the data and extracts the significances of either $\mathrm{NH}$ or IH, is introduced in [7]. First, the Poisson distributions for $n$ observed events $f_{\mathrm{MO}}\left(n ; \mu_{\mathrm{MO}} \mid \delta_{\mathrm{CP}}\right)$ are considered, where $\mu_{\mathrm{MO}}\left(\delta_{\mathrm{CP}}\right)$ are the expected number of events as function of $\delta_{\mathrm{CP}}$. For a specific $n$ the left and right cumulative 


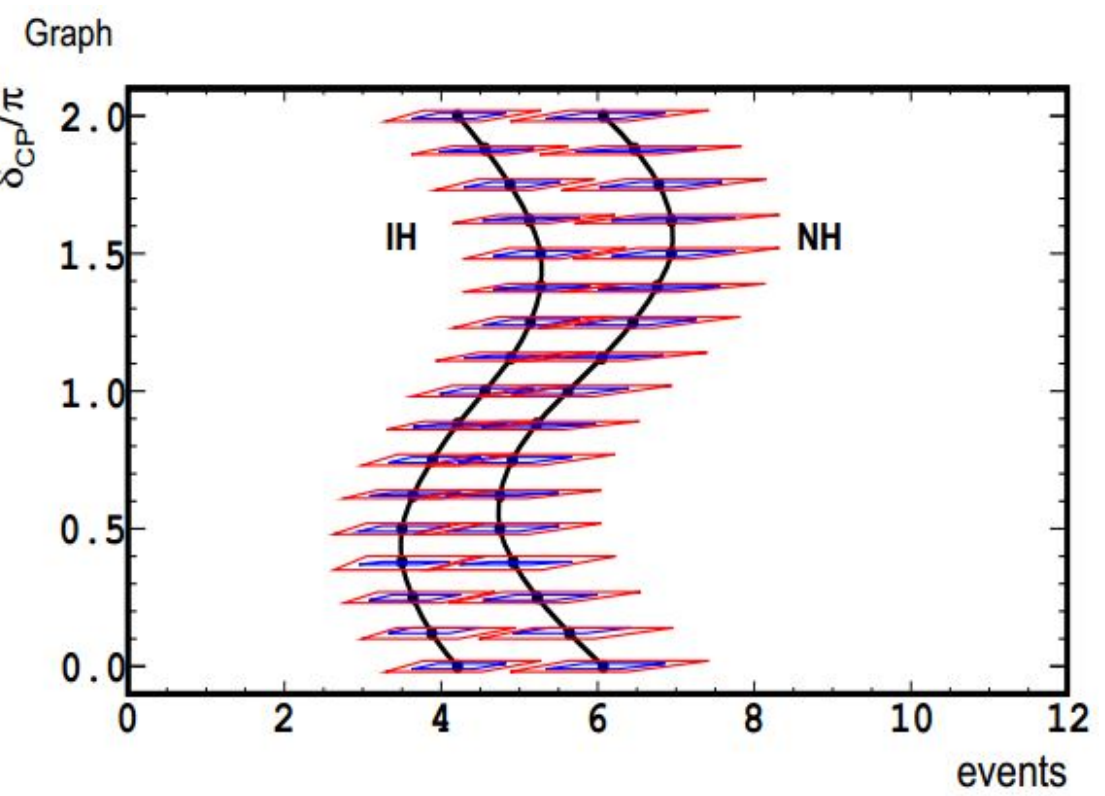

Figure 1: The number of predicted oscillated $v_{\mu} \rightarrow v_{e}$ events plus the expected background is shown in the horizontal axis versus $\delta_{\mathrm{CP}}$ in the vertical axis (the left and right continuous lines for the IH and $\mathrm{NH}$ hypotheses, respectively). The two concentric areas for 16 representative values of $\delta_{\mathrm{CP}}$ spanning its range correspond to the $1 \sigma$ and $2 \sigma$ contours due to the (correlated) $\theta_{23}, \theta_{13}$ uncertainties [1].

functions of $f_{\mathrm{IH}}$ and $f_{\mathrm{NH}}$ are computed and their ratios, $q_{\mathrm{MO}}$, are evaluated. The ratios are similar to the CLs test statistic used for the Higgs discovery $[8,9]$. Since for the $v_{e}$ appearance in the NOvA experiment the number of expected events as function of $\delta_{\mathrm{CP}}$ is asymmetric towards $\mathrm{IH}$ and $\mathrm{NH}$ (less events are expected for $\mathrm{IH}$ than for $\mathrm{NH}$ ), the CLs are defined for either the $\mathrm{IH}$ or the $\mathrm{NH}$ case:

$$
q_{\mathrm{IH}}\left(n, \delta_{\mathrm{CP}}\right)=\frac{\sum_{n_{i}^{\mathrm{IH}} \geq n} f_{\mathrm{IH}}\left(n_{i}^{\mathrm{IH}} ; \mu_{\mathrm{HH}} \mid \delta_{\mathrm{CP}}\right)}{\sum_{n_{i}^{\mathrm{NH}} \geq n} f_{\mathrm{NH}}\left(n_{i}^{\mathrm{NH}} ; \mu_{\mathrm{NH}} \mid \delta_{\mathrm{CP}}\right)} \quad \text { and } \quad q_{\mathrm{NH}}\left(n, \delta_{\mathrm{CP}}\right)=\frac{\sum_{n_{i}^{\mathrm{NH}} \geq n} f_{\mathrm{NH}}\left(n_{i}^{\mathrm{NH}} ; \mu_{\mathrm{NH}} \mid \delta_{\mathrm{CP}}\right)}{\sum_{n_{i}^{\mathrm{IH}} \geq n} f_{\mathrm{IH}}\left(n_{i}^{\mathrm{IH}} ; \mu_{\mathrm{IH}} \mid \delta_{\mathrm{CP}}\right)}
$$

$q_{\mathrm{IH}}$ and $q_{\mathrm{NH}}$ are two discretized random variables comprised to the [0, 1] interval. As $n$ goes to zero $q_{\mathrm{IH}}$ goes to one, while when $n$ increases $q_{\mathrm{IH}}$ asymptotically tends to zero. the variable $q_{\mathrm{NH}}$ behaves the other way around towards $n$. For illustration purpose the behaviours of $f_{\mathrm{MO}}$ and $q_{\mathrm{MO}}$ are shown in Fig. 2 for a typical case $(n=8)$.

The probability mass functions, $P(n)$, of each $q_{\text {мо }}$ have been computed via toy Monte Carlo simulations based either on $f_{\mathrm{IH}}$ (test of $\mathrm{IH}$ against $\mathrm{NH}$ ) or $f_{\mathrm{NH}}$ (test of $\mathrm{NH}$ against $\mathrm{IH}$ ). They are further compared to the observed data $n_{D}$, the number of observed events either in real data or in Monte Carlo simulation, evaluating the p-value probabilities for $n_{D}$ :

$$
p_{\mathrm{IH}}\left(n_{D}, \delta_{\mathrm{CP}}\right)=\sum_{q_{\mathrm{IH}}^{\prime} \leq q_{\mathrm{IH}}\left(n_{D}\right)} P_{\mathrm{IH}}\left(q_{\mathrm{IH}}^{\prime} \mid \delta_{\mathrm{CP}}\right) \quad \text { and } \quad p_{\mathrm{NH}}\left(n_{D}, \delta_{\mathrm{CP}}\right)=\sum_{q_{\mathrm{NH}}^{\prime} \leq q_{\mathrm{NH}}\left(n_{D}\right)} P_{\mathrm{NH}}\left(q_{\mathrm{NH}}^{\prime} \mid \delta_{\mathrm{CP}}\right)
$$



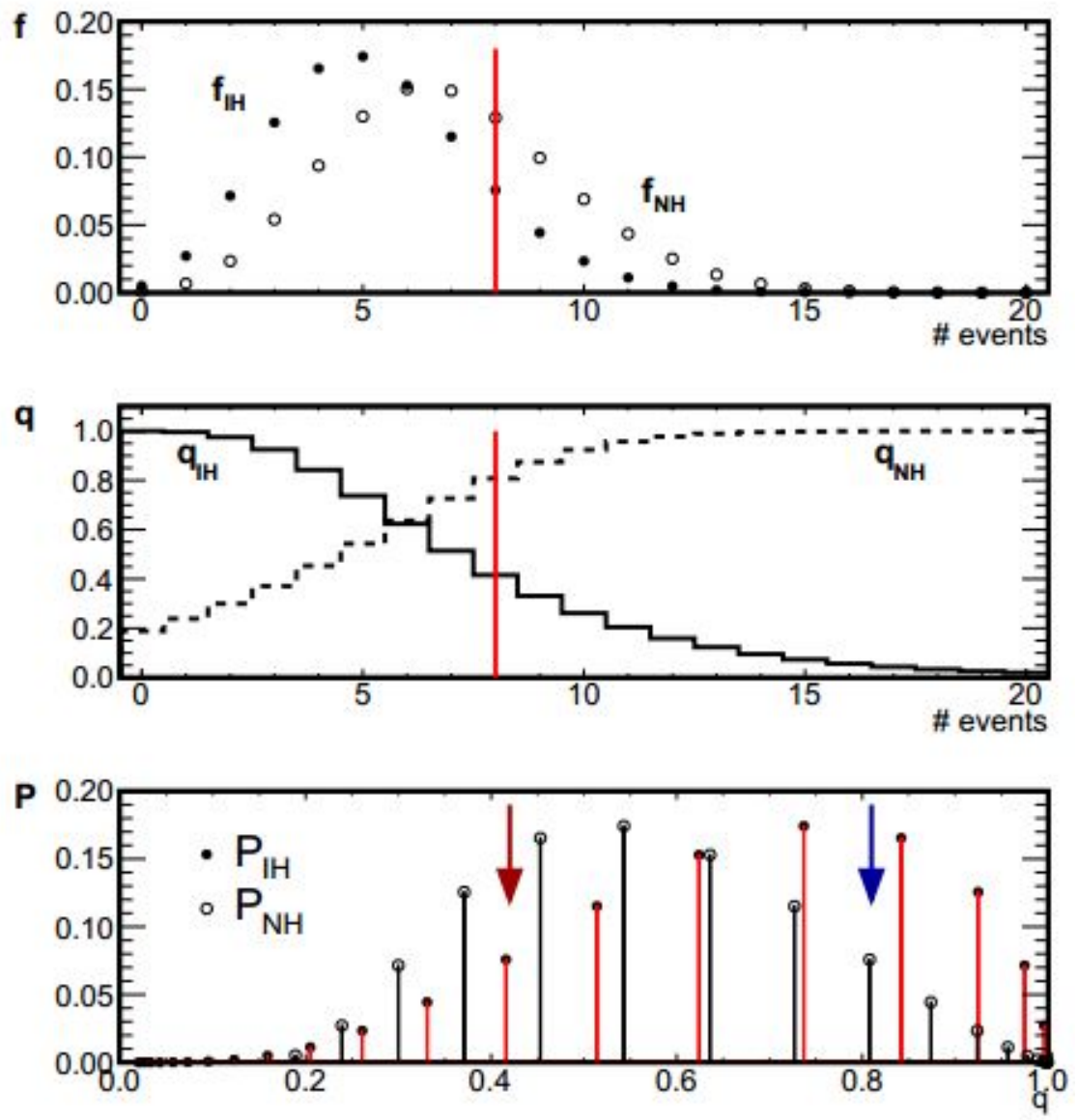

Figure 2: Top: the predicted Poisson distributions of the 2015 NOvA analysis (signal plus background) are shown for IH (full points) and NH (open points), for $\delta_{\mathrm{CP}}=3 / 2 \pi$ and an exposure of $2.74 \times 10^{20}$ p.o.t. The vertical line corresponds to $n=8$. Middle: the corresponding values assumed by $q_{\mathrm{IH}}$ (plain line) and $q_{\mathrm{NH}}$ (dashed line). Bottom: the probability mass functions of $q_{\mathrm{IH}}$ (full points) and $q_{\mathrm{NH}}$ (open points). The arrows indicate the thresholds used to compute $q_{\mathrm{IH}}$ and $q_{\mathrm{NH}}$ for $n=8$.

Finally the significance has been computed with the one-sided option since this corresponds to 0 sigma $(Z=0)$ for $p=50 \%$ that equalizes the $\mathrm{IH}$ and $\mathrm{NH}$ probabilities. Within that choice $Z$ is defined as $Z=\Phi^{-1}(1-p)$, where $\Phi^{-1}$ is the quantile of the standard Gaussian and $Z$ is the number of standard deviations. The uncertainties on $\theta_{23}$ and $\theta_{13}$, as well as the systematic errors, let fluctuate the prediction of the median number of events. These uncertainties have been taken into account using two approaches: A) convolution of the Poisson distributions with assumed Gaussian distributions [10] for the uncertainties on $\theta_{23}, \theta_{13}$ and the systematic errors; B) evaluation of the error bands overlaying the significance, choosing a $\pm \sigma$ variation of the mixing angles and the systematic errors.

With the new method an averaged increase of $0.5 \sigma$ with respect to the standard $\chi^{2}$ minimization is obtained. Worth to note that the increase is not constant but it depends on the discrimination threshold $n_{D}$ and $\delta_{\mathrm{CP}}$ : the gain of the new method in terms of the number of sigma's strongly raises with $n_{D}$ and "favourable" regions of $\delta_{\mathrm{CP}}$. Results are much more promising when the data sample 
increases. For example if a factor 3 in exposure is applied to the 2015 NOvA analysis the rejection of IH can reach the $95 \%$ C.L. in almost the full range of $\delta_{\mathrm{CP}}$, even including the current uncertainties on $\theta_{23}$ and $\theta_{13}$ in a Bayesian way in Fig. 3.

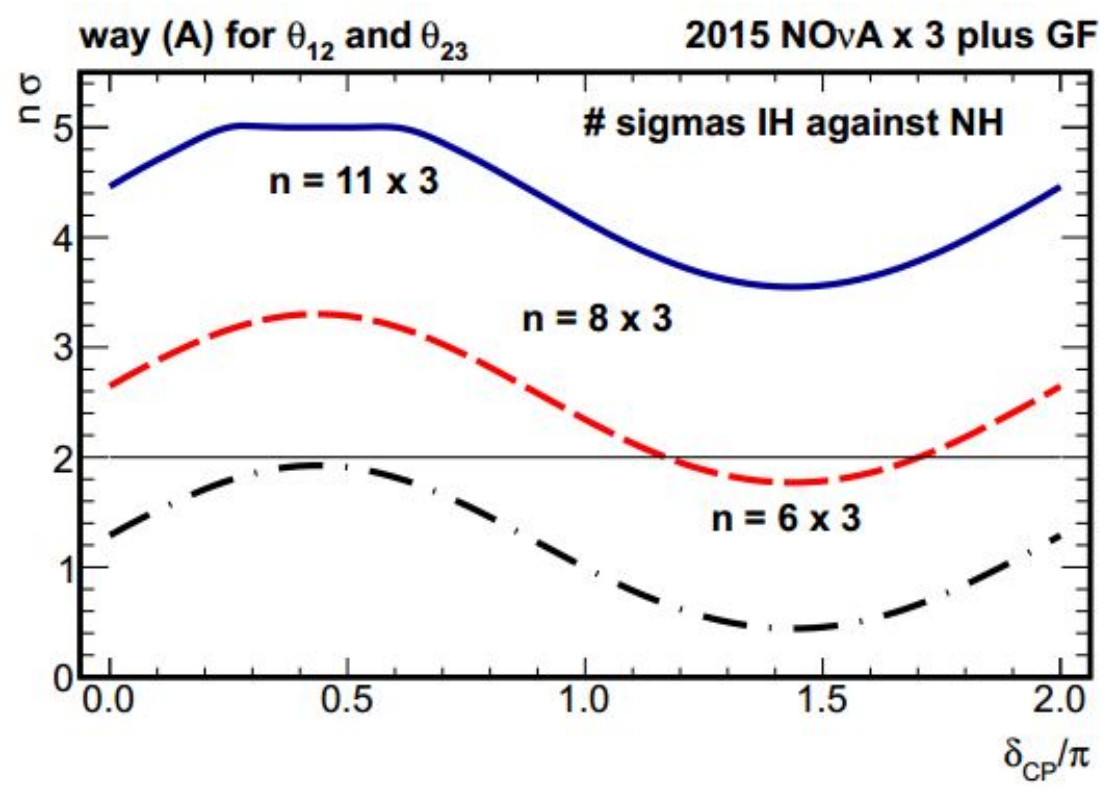

Figure 3: The significance from the the new method in terms of $\sigma$ (one-sided option) is shown for a total exposure of $8.22 \times 10^{20}$ p.o.t. (top), assuming the same efficiency for the signal and the same level of background rejection of 2015 NOvA analysis. The tested hypothesis is IH against NH. The different curves correspond to different possible observations of events, 18, 24 and 33 (signal plus background), as estimated by 2015 NOvA in the $v_{\mu} \rightarrow v_{e}$ channel. The uncertainties on $\theta_{23}$ and $\theta_{13}$ are treated as nuisances (way A).

The new method works better when some statistical fluctuations with respect to the median expectations should be observed. The $95 \%$ C.L. exclusion is obtained for the case $3 \times 8=24$ events that is slightly far from the median expectations (at $\delta_{\mathrm{CP}}=1.5 \pi, 3 \times 5.3=15.8$ and $3 \times 6.9=20.8$ are expected for $\mathrm{IH}$ and $\mathrm{NH}$, respectively). Including the systematic errors, scaled from the 2015 NOvA analysis, the achievement is not spoiled, with about a decrease of 0.3-0.4 $\sigma$ in significance.

NOvA collaboration released new results [11] obtained from a total exposure of $6.05 \times 10^{20}$ p.o.t., a factor 2.2 with regard to the 2015 results. 33 events for $v_{\mu} \rightarrow v_{e}$ appearance production were found. However the background level was highly enhanced (factor 4.5) against an increase of the efficiency of a factor 2.5. By scaling these number to the 2015 analysis and exposure, the 33 events of 2016 corresponds to about 6 events in 2015. That is around the median expectation without an even moderate fluctuation. Any how, applying the new method, the gain in exposure from 2015 to 2016 is sufficient to obtain a first important result: the inverted hierarchy can be excluded at $95 \%$ C.L. in the $\delta_{\mathrm{CP}}$ interval $[0.10 \pi, 0.77 \pi]$.

\section{Conclusions}

The neutrino MO is one of the most relevant questions to be answered in the near future. It is mandatory to explore new ways in statistical analysis, also motivated by the too simple methods 
applied so far, all based on the minimization of the $\chi^{2}$. The new method introduced in [7] is quite promising. In the next few years, working out first the NOvA data sample, it would provide reliable results on the mass hierarchy, within the framework of the three flavours neutrino model. If the future NOvA results would be in line with its 2015 result where some fluctuation from the median expectations were observed, and assuming the three-neutrino oscillation paradigm be true, then the inverted hierarchy could be disproved at 95\% C.L. in the full range of $\delta_{\mathrm{CP}}$. Meanwhile, with the 2017 NOvA data release, the new method allows to exclude IH at 95\% C.L. in the $\delta_{\mathrm{CP}}$ interval $[0.10 \pi, 0.77 \pi]$.

\section{References}

[1] F. Capozzi, E. Lisi, A. Marrone, D. Montanino and A. Palazzo, "Neutrino masses and mixings: Status of known and unknown $3 v$ parameters," Nucl. Phys. B 908 (2016) 218 doi:10.1016/j.nuclphysb.2016.02.016 [arXiv:1601.07777 [hep-ph]].

[2] E. Ciuffoli, J. Evslin and X. Zhang, "Confidence in a neutrino mass hierarchy determination," JHEP 1401 (2014) 095 doi:10.1007/JHEP01(2014)095 [arXiv:1305.5150 [hep-ph]].

[3] M. Blennow, "On the Bayesian approach to neutrino mass ordering," JHEP 1401 (2014) 139 doi:10.1007/JHEP01(2014)139 [arXiv:1311.3183 [hep-ph]].

[4] P. Adamson et al. [NOvA Collaboration], "First measurement of electron neutrino appearance in NOvA,” Phys. Rev. Lett. 116 (2016) no.15, 151806 doi:10.1103/PhysRevLett.116.151806 [arXiv:1601.05022 [hep-ex]].

[5] P. Huber, M. Lindner and W. Winter, "Simulation of long-baseline neutrino oscillation experiments with GLoBES (General Long Baseline Experiment Simulator)," Comput. Phys. Commun. 167 (2005) 195 doi:10.1016/j.cpc.2005.01.003 [hep-ph/0407333].

[6] P. Huber, J. Kopp, M. Lindner, M. Rolinec and W. Winter, "New features in the simulation of neutrino oscillation experiments with GLoBES 3.0: General Long Baseline Experiment Simulator," Comput. Phys. Commun. 177 (2007) 432 doi:10.1016/j.cpc.2007.05.004 [hep-ph/0701187].

[7] L. Stanco, S. Dusini and M. Tenti, "Determination of the neutrino mass hierarchy with a new statistical method," Phys. Rev. D 95 (2017) no.5, 053002 doi:10.1103/PhysRevD.95.053002 [arXiv:1606.09454 [hep-ph]].

[8] A. L. Read, "Presentation of search results: The CL(s) technique," J. Phys. G 28 (2002) 2693. doi:10.1088/0954-3899/28/10/313

[9] G. Cowan, K. Cranmer, E. Gross and O. Vitells, "Asymptotic formulae for likelihood-based tests of new physics," Eur. Phys. J. C 71 (2011) 1554 Erratum: [Eur. Phys. J. C 73 (2013) 2501] doi:10.1140/epjc/s10052-011-1554-0, 10.1140/epjc/s10052-013-2501-z [arXiv:1007.1727 [physics.data-an]].

[10] R. D. Cousins and V. L. Highland, "Incorporating systematic uncertainties into an upper limit," Nucl. Instrum. Meth. A 320 (1992) 331. doi:10.1016/0168-9002(92)90794-5

[11] P. Adamson et al. [NOvA Collaboration], "Constraints on Oscillation Parameters from $v_{e}$ Appearance and $v_{\mu}$ Disappearance in NOvA," Phys. Rev. Lett. 118 (2017) no.23, 231801

doi:10.1103/PhysRevLett.118.231801 [arXiv:1703.03328 [hep-ex]]. 